\title{
Charge carrier transport and electroluminescence in atomic layer deposited poly-GaN/c-Si heterojunction diodes
}

\author{
Gaurav Gupta, ${ }^{1, a)}$ Sourish Banerjee, ${ }^{1}$ Satadal Dutta, ${ }^{1}$ Antonius A.I. Aarnink, ${ }^{1}$ Jurriaan Schmitz, ${ }^{1}$ Alexey \\ Y.Kovalgin, ${ }^{1}$ and Raymond J.E. Hueting ${ }^{1}$ \\ University of Twente, Enschede, The Netherlands
}

(Dated: 15 September 2018)

In this work, we study the charge carrier transport and electroluminescence (EL) in thin-film polycrystalline (poly-) GaN/c-Si heterojunction diodes realized using a plasma enhanced atomic layer deposition (PE-ALD) process. The fabricated poly-GaN/p-Si diode with a native oxide at the interface showed a rectifying behavior $\left(I_{\text {on }} / I_{\text {off }}\right.$ ratio $\sim 10^{3}$ at $\left.\pm 3 \mathrm{~V}\right)$ with current-voltage characteristics reaching an ideality factor $n$ of $\sim 5.17$. The areal $\left(J_{\mathrm{a}}\right)$ and peripheral $\left(J_{\mathrm{p}}\right)$ components of the current density were extracted and their temperature dependencies were studied. The space charge limited current (SCLC) in the presence of traps is identified as the dominant carrier transport mechanism for $J_{\mathrm{a}}$ in forward bias. An effective trap density of $4.6 \times 10^{17} / \mathrm{cm}^{3}$ at a trap energy level of $0.13 \mathrm{eV}$ below the GaN conduction band minimum was estimated by analyzing $J_{\mathrm{a}}$. Other basic electrical properties of the material such as free carrier concentration, density of states in the conduction band, electron mobility and dielectric relaxation time were also determined from the current-voltage analysis in the SCLC regime. Further, infrared EL corresponding to the Si bandgap was observed from the fabricated diodes. The observed EL intensity from the GaN/p-Si heterojunction diode is $\sim 3$ orders of magnitude higher as compared to the conventional Si only counterpart. The enhanced infrared light emission is attributed to the improved injector efficiency of the GaN/Si diode because of the wide bandgap of the poly-GaN layer and resulting band discontinuity at the GaN/Si interface.

Keywords: ALD, GaN, Si, heterojunction, infrared emission, space charge limited current, polycrystalline, thin film.

\section{INTRODUCTION}

Gallium Nitride (GaN) is an attractive semiconductor for both electronic and optoelectronic applications because of its wide and direct bandgap, high breakdown field and stability at high temperature ${ }^{1-3}$. The relatively high substrate cost for GaN based devices may be tackled with GaN-on-Si substrates. These additionally allow the monolithic integration of GaN devices with Si platform technolog $\mathrm{y}^{4-6}$. However, when monocrystalline GaN is to be grown on silicon, a several-micrometer thick buffer layer is required ${ }^{4,7}$ which makes it difficult to form a platform technology combining silicon devices with GaN devices on the same chip. A potential alternative approach is to grow thin-film polycrystalline GaN on silicon. Compared to thick film heteroepitaxy, polycrystalline films bring important fabrication advantages such as lower cost, a lower bill-of-materials and a lower thermal budget. Still, polycrystalline materials generally do not reach the same quality in electrical performance as their monocrystalline counterparts. It is therefore the purpose of this work to investigate electrical properties of polycrystalline GaN grown on monocrystalline silicon substrates.

Among the several approaches to grow thin polycrystalline GaN films, atomic layer deposition (ALD) is an attractive solution for applications where excellent waferlevel uniformity, a critical layer thickness control is re-

\footnotetext{
a)G.Gupta@utwente.nl
}

quired, and complex device topologies are involved ${ }^{8,9}$. An additional advantage of ALD is that it allows to reduce the deposition temperature of many processes ${ }^{10}$. Recently, ALD grown GaN devices such as thin-film transistors have been successfully demonstrated ${ }^{11-13}$ with a reasonable performance considering their polycrystalline nature. However so far there have been no reports on vertical ALD-GaN-on-Si heterojunctions in active devices. With this motivation, we study ALD grown thin-film polycrystalline GaN/Si heterojunction diodes and investigate their charge carrier transport and optical properties.

\section{EXPERIMENTAL}

A (111) oriented p-type Si wafer with a background doping concentration of $10^{15} \mathrm{~cm}^{-3}$ was used to fabricate the $\mathrm{GaN} / \mathrm{p}$-Si heterojunction diodes. The native oxide present on the surface of the Si wafer was not removed for this experiment. A thin layer of polycrystalline GaN on Si substrate was deposited using a plasma-enhanced ALD process, in a remote-plasma Picosun R-200 reactor. The precursors were trimethylgallium (TMG) for $\mathrm{Ga}$ and ammonia (NH3) for $\mathrm{N}$. The depositions were done at 400 ${ }^{\circ} \mathrm{C}$ temperature. The growth of the layers were monitored in real-time using an in-situ spectroscopic ellipsometer (J.A. Woollam Co. M-2000, operating between 245.8 - $1688.5 \mathrm{~nm}$ wavelength). All the polycrystalline GaN samples studied in this work were of $36 \pm 3 \mathrm{~nm}$ thickness. 
Post deposition, the GaN layers were characterized by X-ray photoelectron spectroscopy (XPS, Phi Quantera SXM) for determining the layer composition and by $\theta-2 \theta$-and grazing incidence angle X-ray diffraction (GIXRD, Panalytical XPert Powder) for determining the layer crystallinity. The layer thicknesses that were obtained from the ellipsometer in-situ monitoring were additionally verified by a transmission electron microscope (TEM, Philips CM300ST-FEG).

$\mathrm{GaN} / \mathrm{Si}$ heterojunction diodes with $\mathrm{Al}$ metal electrodes (i.e., $\mathrm{Al}$ as a top electrode) were then fabricated using a standard photolithography process. The schematic of experimentally realized device is shown in the inset of Fig. 2. All the electrical measurements were performed using a Keithley 4200 semiconductor parameter analyser that was equipped with a temperature-controlled chuck. The Si substrate was utilized as the bottom contact. Optical measurements were performed using a cooled InGaAs detector based camera (XEVA-320 from Xenics) for capturing the IR micrographs. For measuring the emission spectra, an Avaspec UV-Vis/NIR spectrometer from Avantes was used. Further, an off-chip Si photodiode ${ }^{14}$ was utilized for photocurrent measurements.

\section{RESULTS AND DISCUSSION}

\section{A. Material characterization of the GaN layers}
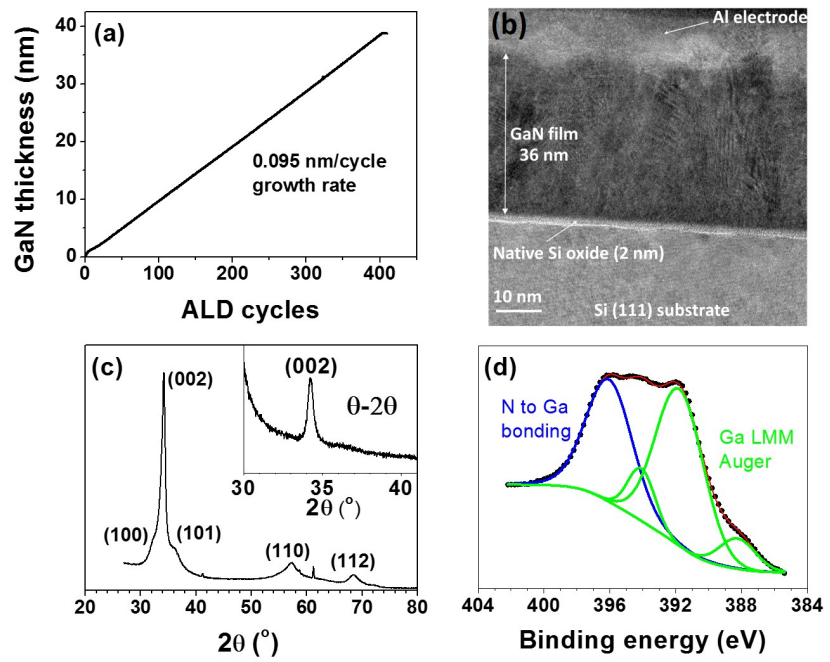

FIG. 1. (a) GaN growth on a Si substrate monitored in real time by in-situ SE, (b) TEM image of a sample cross-section (c) GIXRD scan revealing polycrystalline wurtzitic nature of grown sample. Inset: $\theta-2 \theta$ XRD scan showing strong reflection from the (002) wurtzitic planes. (d) XPS spectrum of the $\mathrm{N}$ 1s (in blue) and Auger spectra of the Ga LMM (in green) as obtained after depth-profiling in the sample. The acquired data points are shown in black.

The deposition of polycrystalline (poly-) GaN layers was monitored in real time using in-situ spectroscopic ellipsometry (SE) (Fig. 1(a)). The layers had a growth rate of $\sim 0.095 \mathrm{~nm} /$ cycle. Since the layer thicknesses were extracted using an optical model ${ }^{15}$, it was necessary to validate the obtained thickness values. This was done with the help of TEM imaging (Fig. 1(b)). Both SE and TEM revealed similar thickness values, i.e., $\sim 36 \mathrm{~nm}$ within an accuracy of $\pm 3 \mathrm{~nm}$.

The TEM image of Fig. 1(b) shows the cross-section of the fabricated device (i.e. Al electrode on GaN/Native Oxide/Si wafer). The GaN layer reveals vertical (columnar) growth at several regions. Fast Fourier Transform $(\mathrm{FFT})$ at several regions in the layer revealed d-spacing values predominantly associated with the (002) planes of hexagonal (wurtzitic) GaN. Other wurtzitic crystal planes such as (100) and (101) were also observed in small amounts, indicating the polycrystalline nature of the material.

The polycrystalline structure was reconfirmed with the help of a GIXRD scan (Fig. 1(c)) taken at an incidence angle of $1^{\circ}$. The diffractogram confirmed the wurtzitic structure of the layer and also shows a strong (002) peak, reflecting the abundance of this crystal plane in the polycrystalline layer. Since this is a grazing incidence scan, the reflection comes from tilted (002) planes. A $\theta-2 \theta$ XRD scan of the layer (see inset Fig. 1(c)) again showed a strong (002) reflection with an almost-complete absence of other crystal planes. This again indicates that a significant number of (002) crystal domains are stacked parallel to the substrate which is in accordance with the observation of columnar growth from TEM.

The average grain size of the (002) crystal plane from the diffractogram was estimated to be $9.8 \mathrm{~nm}$. This value is slightly higher compared to that observed from TEM images, which showed grain sizes between $\sim 5-9 \mathrm{~nm}$.

The nature of chemical bonding in the layers was determined from XPS, which was equipped with an Al$k \alpha$ monochromatic X-ray source. Additionally, sputter depth profiling with $1 \mathrm{keV} \mathrm{Ar}^{+}$was done in order to obtain the composition and the bonding information as a function of layer thickness. As an example, Fig. 1(d) (in blue) shows the $\mathrm{N}$ 1s photoelectron spectrum from the bulk of the layer. This spectrum is in fact convoluted with the Ga LMM Auger triplet (in green). The deconvolution reveals the position of the N-Ga bond at $396.2 \mathrm{eV}$ which is in the vicinity of the literature reported value $^{16}$ of $397 \mathrm{eV}$.

\section{B. Electrical Measurements}

The $I-V$ characteristics of the GaN/p-Si diode with native oxide at $298 \mathrm{~K}$ (Fig. 2) show a rectifying behavior with an $I_{\text {on }} / I_{\text {off }}$ ratio $\sim 10^{3}$ at $\pm 3 \mathrm{~V}$. However the semi-logarithmic $I-V$ plot shows a high ideality factor $n \sim 5.17$. An ideality factor of much higher than two indicates a physical transport mechanism other than thermionic emission or diffusion $(n=1)$ and space charge region recombination $(n \leq 2)$. The GaN layer could limit 
the conduction by bulk controlled processes such as space charge limited current (SCLC) or Poole-Frenkel mechanism. Alternatively, the conduction could be limited by interface controlled processes at the GaN/Si heterojunction such as thermionic emission or tunneling.

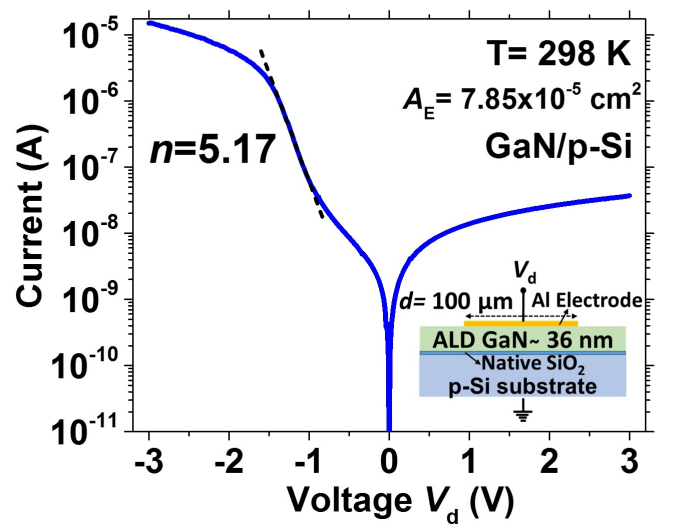

FIG. 2. $I-V$ characteristics of fabricated $\mathrm{GaN} / \mathrm{p}-\mathrm{Si}$ vertical diode with native oxide at $T=298 \mathrm{~K}$. Inset: schematic cross-section of the experimental device with circular electrode (area $A_{\mathrm{E}}$ ) of diameter $d=100 \mu \mathrm{m}$.

Fig. 3 shows a simulated energy band diagram of the GaN/p-Si heterojunction in which the Anderson's model or electron affinity rule was used ${ }^{17}$. Here, the electronaffinity of GaN was assumed to be $3.3 \mathrm{eV}$ which has been reported before both for crystalline $\mathrm{GaN}^{18,19}$ and for polycrystalline $\mathrm{GaN}^{20,21}$. The large discontinuity in the valence band $\left(\Delta E_{\mathrm{v}}=1.58 \mathrm{eV}\right)$ as compared to conduction band discontinuity $\left(\Delta E_{\mathrm{c}}=0.7 \mathrm{eV}\right)$ makes it less likely for holes to participate in the conduction process, at least at lower voltages $(<1.58 \mathrm{~V})$. Therefore it is very likely that only electrons will cause conduction.

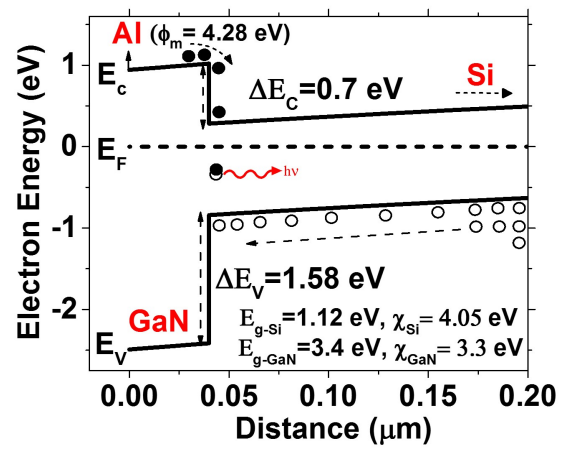

FIG. 3. TCAD ${ }^{17}$ simulated energy band diagram of the $\mathrm{GaN} / \mathrm{p}$-Si heterojunction diode at thermal equilibrium. The distance is relative to the top $\mathrm{Al}$ electrode. The GaN layer thickness is $40 \mathrm{~nm}$ and thickness of Si substrate is $525 \mu \mathrm{m}$. Filled circles: electrons. Open circles: holes.

To further analyze the transport mechanism, we first separated the areal $\left(J_{\mathrm{a}}\right)$ from the peripheral $\left(J_{\mathrm{p}}\right)$ current density components via $J-V$ measurement on devices with different perimeter-to-area $(P / A)$ ratio electrodes as shown in Fig. 4. Both $J_{\mathrm{a}}$ and $J_{\mathrm{p}}$ are rectifying in nature.

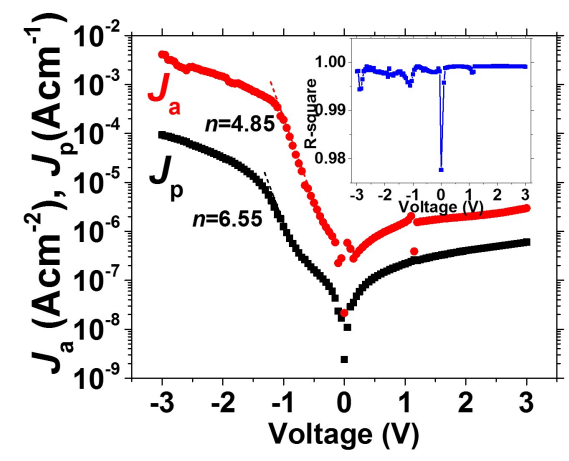

FIG. 4. Extracted $J_{\mathrm{a}}$ and $J_{\mathrm{p}}$ via $J-V$ measurement on devices with different perimeter-to-area $(P / A)$ ratio electrodes using the relationship $J=J_{\mathrm{a}}+(P / A) J_{\mathrm{p}}$, where $J$ total current density. The slope of $J$ vs $P / A$ curve yields $J_{\mathrm{p}}$ while the intercept results in $J_{\mathrm{a}}$. Inset: R-square residual from the least-square fitting method. The R-square value of $\sim 1$ throughout the measured voltage range indicates good linear fitting and therefore uniform current scaling.

The extracted $J_{\mathrm{a}}$ with a high ideality factor could be explained in terms of space charge limited current (SCLC) ${ }^{22,23}$ which is a typical conduction mechanism for thin insulating films or lowly doped wide bandgap semiconductors where only a single type of charge carrier participate in the conduction process. This is very likely the case in our devices where no doping was introduced in the GaN layer and conduction is possibly via electrons at lower voltages. The trap-mediated SCLC has been reported before for GaN based films and nanostructures ${ }^{24-27}$, GaN/Si heterojunctions ${ }^{28-30}$ as well as for other wide bandgap semiconductor based heterojunctions such as $\mathrm{ZnO} / \mathrm{Si}^{31,32}$ and $\mathrm{ZnO} / \mathrm{GaN}^{33}$.

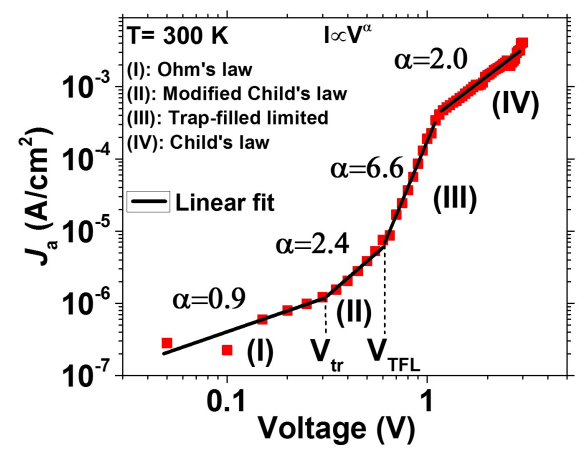

FIG. 5. $\log (J)-\log (V)$ plot of the extracted $J_{\mathrm{a}}$ in the forward bias region.

We analyzed the observed $J_{\mathrm{a}}-V$ behavior in terms 
of a single type of carrier (electrons) SCLC model ${ }^{22,23}$ in the presence of a single discrete trap level at energy $E_{\mathrm{t}}$ below the conduction band edge. The $\log \left(J_{\mathrm{a}}\right)-\log (V)$ plot of the GaN/p-Si diode, see Fig. 5, shows four linear regimes with different slopes $(\alpha)$ which are separated by a transition voltage $V_{\mathrm{tr}}$ and a trap-filled limit (TFL) voltage $V_{\mathrm{TFL}}$. The $J-V$ equations for SCLC in the presence of a single shallow trap level are described as follows ${ }^{23}$ :

$$
\begin{gathered}
J_{\mathrm{ohm}}=q n_{0} \mu \frac{V}{d}, \\
J_{\text {Child(trap) }}=\frac{9 \mu \epsilon_{\mathrm{r}} \epsilon_{0} \theta V^{2}}{8 d^{3}}, \\
J_{\text {Child }}=\frac{9 \mu \epsilon_{\mathrm{r}} \epsilon_{0} V^{2}}{8 d^{3}}, \\
V_{\mathrm{tr}}=\frac{8 q n_{0} d^{2}}{9 \epsilon_{\mathrm{r}} \epsilon_{0} \theta}, \\
V_{\mathrm{TFL}}=\frac{q N_{\mathrm{t}} d^{2}}{2 \epsilon_{\mathrm{r}} \epsilon_{0}}, \\
\theta=\frac{N_{\mathrm{C}}}{g_{\mathrm{n}} N_{\mathrm{t}}} \exp \left(\frac{E_{\mathrm{C}}-E_{\mathrm{t}}}{k T}\right), \\
\tau_{\mathrm{c}}=\frac{d^{2}}{\mu V_{\mathrm{tr}}},
\end{gathered}
$$

where $q$ is the elementary charge, $k$ is the Boltzmann constant, $\epsilon_{0}$ is the permittivity of vacuum, $d$ is the thickness of the insulator or space charge region, $\epsilon_{\mathrm{r}}$ is the relative permittivity of the medium, $\mu$ is the electron mobility, $E_{\mathrm{C}}$ is the position of conduction band edge, $g_{\mathrm{n}}$ is the degeneracy of the states in the conduction band $(\sim 2)^{34}, N_{\mathrm{C}}$ is the conduction band effective density of states and $N_{\mathrm{t}}$ is the effective trap density of the medium.

At low bias $\left(0<V<V_{\mathrm{tr}}\right.$, region I), there is ohmic conduction (Eq.(1)) with $\alpha \sim 1$ where the thermally generated free carrier density $\left(n_{0}\right)$ is larger than the injected carrier density $(n)$. In this region, the carrier transit time $\left(\tau_{\mathrm{c}}\right)$ is greater than the material dielectric relaxation time $\left(\tau_{\mathrm{d}}\right)$ and material remains in quasi-neutral state ${ }^{34}$. At $V=V_{\mathrm{tr}}$, a transition from ohmic conduction to SCLC occurs as $n$ becomes larger than $n_{0}$ and $\tau_{\mathrm{c}}$ just becomes equal to $\tau_{\mathrm{d}}$.

In region II ( $\left.V_{\mathrm{tr}}<V<V_{\mathrm{TFL}}\right)$ the electron quasi-Fermi level $\left(E_{\mathrm{Fn}}\right)$, which is a function of injected charge in case of extrinsic type conduction, is still below the shallow trap energy level $E_{\mathrm{t}}$. In this regime, most of the injected charge does not contribute to the current and part of it
TABLE I. Extracted electrical properties of poly-GaN material at $300 \mathrm{~K}$ from the $J_{\mathrm{a}}-V$ analysis.

\begin{tabular}{|l|c|}
\hline Properties & Values \\
\hline $\begin{array}{l}\text { Transition voltage from ohmic to SCLC } \\
\text { regime, } V_{\mathrm{tr}}\end{array}$ & $0.31 \mathrm{~V}$ \\
\hline Trap-filled-limited voltage, $V_{\mathrm{TFL}}$ & $0.61 \mathrm{~V}$ \\
\hline Effective trap density, $N_{\mathrm{t}}$ & $4.6 \times 10^{17} / \mathrm{cm}^{3}$ \\
\hline Trap energy level, $E_{\mathrm{C}}-E_{\mathrm{t}}$ & $0.13 \mathrm{eV}$ \\
\hline Free carrier concentration, $n_{0}$ & $4.4 \times 10^{15} / \mathrm{cm}^{3}$ \\
\hline Fermi level, $E_{\mathrm{C}}-E_{\mathrm{Fn}}$ & $0.18 \mathrm{eV}$ \\
\hline $\begin{array}{l}\text { Conduction band effective density } \text { of } \\
\text { states, } N_{\mathrm{C}}\end{array}$ & $4.6 \times 10^{18} / \mathrm{cm}^{3}$ \\
\hline Ratio of free carriers to total carries, $\theta$ & 0.03 \\
\hline Electron mobility, $\mu$ & $1.7 \times 10^{-8} \mathrm{~cm}^{2} / \mathrm{V}-\mathrm{s}$ \\
\hline Dielectric relaxation time, $\tau_{\mathrm{d}}$ & $2.4 \times 10^{-3} \mathrm{~s}$ \\
\hline
\end{tabular}

occupies the traps. Further, the fraction $(\theta)$ of the free charge to the total charge (free and trapped charge) remains constant and doesn't vary with the applied voltage as long as $E_{\mathrm{Fn}}$ is below $E_{\mathrm{t}}{ }^{23}$. The conduction (in region II) follows Child's law however with current density reduced by a factor $\theta$ (Eq.(2)).

Further increasing the injected free carrier density by biasing a higher voltage, moves $E_{\mathrm{fn}}$ further up and eventually at $V=V_{\mathrm{TFL}}, E_{\mathrm{Fn}}$ just passes over $E_{\mathrm{t}}$. Thus, $V_{\text {TFL }}$ is defined as the threshold voltage required to fill the traps. Shortly beyond $V_{\mathrm{TFL}}$, i.e. region III, a steep rise in the current ( $\alpha=6.6)$ occurs as it rapidly recovers from its low trap-limited value to a high trap-free SCLC value. Therefore region III is the transition from a trapped $I-V$ behavior to a trap-free behavior. Finally beyond this transition region, when all traps are completely filled, they no longer affect the charge injection. Hereafter, any injected charge fully contributes to the current (region IV) and an ideal trap-free square law ( $\alpha=2.06$ ) is followed (Eq.(3)).

Using Eqs.(1)-(7) ${ }^{23}$ and following the methodology as also previously described by Chiu et al. ${ }^{34}$, we extracted the basic electrical properties of the poly-GaN material, summarized in Table I. For the calculations, we used $d=36 \mathrm{~nm}$ (GaN thickness) and the literature reported value of $\epsilon_{\mathrm{r}}=8.9$ for $\mathrm{GaN}^{35}$. $V_{\mathrm{tr}}=0.31 \mathrm{~V}$ and $V_{\mathrm{TFL}}=0.61 \mathrm{~V}$ were estimated from Fig. 5. Further, we used temperature dependent measurements (see Fig. 6(a)) to estimate the effective $E_{\mathrm{t}}$ from the Arrhenius plot. The energy levels $E_{\mathrm{Fn}}$ and $E_{\mathrm{t}}$ are relative to $E_{\mathrm{C}}$.

The Arrhenius plot of the $J_{\mathrm{a}}$ at a range of voltages in the forward bias is shown in Fig. 6(b) indicating a ratelimited thermally activated process $\left(J_{\mathrm{a}} \propto \exp \left(-E_{\mathrm{a}} / k T\right)\right)$ where $E_{\mathrm{a}}$ is the thermal activation energy. The estimated $E_{\mathrm{a}}$ is constant throughout the measured temperature range. This also indicates that a single trap level is dominant over the statistics of majority carriers throughout the measured temperature range ${ }^{36}$. We also observed no significant variation $(\sim k T)$ in $E_{\mathrm{a}}$ from ohmic conduction to SCLC regime at different voltage ranges. According to Roberts and Schmidlin ${ }^{36}$, this suggests that con- 

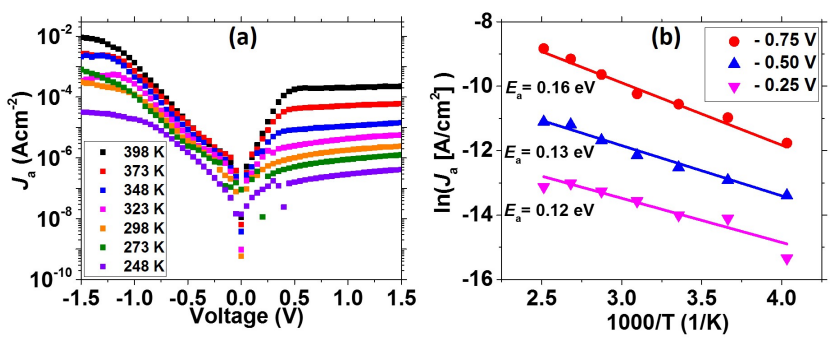

FIG. 6. (a) Temperature dependent $J_{\mathrm{a}}-V$ characteristics of the fabricated $\mathrm{Al} / \mathrm{GaN} / \mathrm{p}-\mathrm{Si}$ diode. (b) Arrhenius plot of the $J_{\mathrm{a}}$ for various forward bias levels.

duction is largely extrinsic in nature, as also expected in wide bandgap materials. The estimated $E_{\mathrm{a}}$ from Arrhenius plot can then be interpreted as the dominant trap energy level $E_{\mathrm{t}}$ as long as $\theta \ll 1$. Hence we estimated $E_{t}=0.13 \mathrm{eV} \pm 0.0259 \mathrm{eV}$ below $E_{\mathrm{c}}$ from the extracted $E_{\mathrm{a}}$ in the trap filling region (II) of the $\log \left(J_{\mathrm{a}}\right)-\log (V)$ curve (Fig. 5). In this region, $E_{\mathrm{Fn}}$ lies below the $E_{\mathrm{t}}$ and therefore $\theta$ which contains the $E_{\mathrm{a}}$ for $J$, can be described by Eq. (6).

It should however be noted that Eq. (6) is only accurate when $\theta \ll 1$ and therefore the interpretation of $E_{\mathrm{a}}$ from the Arrhenius plot as $E_{\mathrm{t}}$ may be erroneous otherwise. A more generalized expression for $\theta$ as given by Eq. $(8)^{23}$ offers better insight into the interpretation of Arrhenius plot.

$$
\theta^{\prime}=\frac{N_{\mathrm{C}} \cdot \exp \left[\frac{\left(E_{\mathrm{C}}-E_{\mathrm{Fn}}\right)}{k T}\right]}{N_{\mathrm{C}} \cdot \exp \left[\frac{\left(E_{\mathrm{C}}-E_{\mathrm{Fn}}\right)}{k T}\right]+\left(\frac{N_{\mathrm{t}}}{1+\left(1 / g_{\mathrm{n}}\right) \cdot \exp \left[\frac{-\left(E_{\mathrm{t}}-E_{\mathrm{Fn}}\right)}{k T}\right]}\right)}
$$

From Eq. (8) various cases depending on the relative positions of $E_{\mathrm{Fn}}$ and $E_{\mathrm{t}}$ within the bandgap can be analyzed. We investigated the impact of Eq. (8) on our results further by inserting the estimated $E_{\mathrm{t}}, N_{\mathrm{C}}$ and $E_{\mathrm{Fn}}$ values from Table I there and varying the $N_{\mathrm{t}}$ from $10^{14}$ till $10^{19} / \mathrm{cm}^{3}$. The modeled Arrhenius plot along with extracted $E_{\mathrm{a}}$ for different $N_{\mathrm{t}}$ is shown in Fig. 7 . We noted that the extracted $E_{\text {a }}$ values agree with given $E_{\mathrm{t}}$ only in a region where $N_{\mathrm{t}} \sim N_{\mathrm{C}}$. Since in our case, $N_{\mathrm{t}}$ is expected to be very high because of the polycrystalline nature of the film, as also reported before ${ }^{24}$, the measured $E_{\mathrm{a}}$ corresponds to $E_{\mathrm{t}}$.

Further, we investigated the role of any other possible conduction mechanism in our diodes. It is possible that the steep slope region in the mid-voltage range $(-1<V<-0.5 \mathrm{~V})$ in Fig. 5 could also be partly originating from the recombination tunneling mechanism ${ }^{37}$. This type of conduction mechanism has been reported before for wide bandgap heterojunctions ${ }^{31,32}$ in the presence of deep level traps. For a single step tunneling recombination pathway, where the electrons from the conduction band of an n-type wide band gap material falls
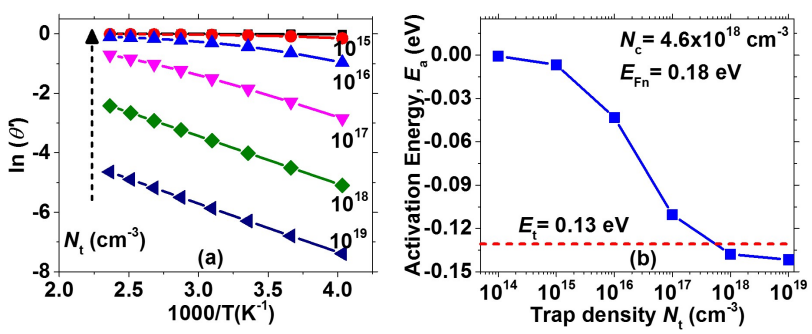

FIG. 7. (a) Modeled Arrhenius plot of $\theta^{\prime}$ using Eq. (8) for various $N_{\mathrm{t}}$. (b) Estimated $E_{\mathrm{a}}$ against $N_{\mathrm{t}}$ for a fixed $E_{\mathrm{t}}=0.13$ $\mathrm{eV}$.

into the empty deep level trap, and subsequently tunnel into the valence band of the p-type material, the forward current density is described as $J=C_{1} N_{\mathrm{t}} \exp (B \cdot V)$ where $C_{1}$ is a constant and $N_{\mathrm{t}}$ is the trap density ${ }^{37}$. The temperature independent exponential pre-factor $B$ is given $\mathrm{as}^{37}$ :

$$
B=(8 \pi / 3 h)\left(m_{\mathrm{h}}^{*} \epsilon_{\mathrm{s}}\right)^{1 / 2} N_{\mathrm{D}} /\left[N_{\mathrm{A}}^{1 / 2}\left(N_{\mathrm{A}}+N_{\mathrm{D}}\right)\right],
$$

where $m_{\mathrm{h}}^{*}$ is the hole effective mass, $\epsilon_{\mathrm{s}}$ is the dielectric constant of the wide band gap material, $N_{\mathrm{D}}$ and $N_{\mathrm{A}}$ are donor and acceptor concentration respectively and $h$ is Planck's constant. The estimated $B$ value $\left(\sim 7.3 \mathrm{~V}^{-1}\right)$ from Fig. 6(a) via curve fitting is temperature independent in the said voltage range. However $J_{\mathrm{a}}$ does show a temperature dependency in the same range unlike expected for the tunneling mechanism ${ }^{37}$. This suggests that recombination tunneling is not the dominant mechanism there.

Other related trap assisted tunneling (TAT) processes such as two-step TAT ${ }^{38,39}$ or even multi-step TAT $^{40}$ are also shown to have a weak temperature dependency. However our $J_{\mathrm{a}}-V-T$ characteristics clearly suggest a rate-limited thermally activated process (Fig. 6(b)). Therefore the TAT current contribution to the forward $J_{\mathrm{a}}$, though possibly present, is not significant and consequently does not affect our SCLC analysis.

We also considered the possibility of Poole-Frenkel type of conduction in our diodes ${ }^{41,42}$. The $\ln \left(J_{\mathrm{a}} / V\right)$ vs. $\sqrt{V}$ plot showed a straight line in the mid voltage range in the forward bias region as expected for Poole-Frenkel type conduction. However, the decrease in its slope with increase in temperature, as also expected for Poole-Frenkel conduction, was not manifested in our data. In addition, $E_{\mathrm{a}}$ also did not decrease with the applied bias unlike in case of Poole-Frenkel conduction where the applied field reduces the effective trap ionization level. Therefore it appears that Poole-Frenkel conduction is not the dominant transport mechanism in our diodes as well.

Our $J_{\mathrm{a}}-V$ analysis along with its temperature dependency strongly suggest the single level shallow trap mediated SCLC as the dominant mechanism. As a san- 
ity check on the proposed SCLC model, we also independently estimated the $N_{\mathrm{t}}$ value from the intercept of the Arrhenius plot (Fig. 6(b)) using Eq. (2) and assuming $N_{\mathrm{c}} \sim 1.2 \times 10^{18} / \mathrm{cm}^{3}$ for $\mathrm{GaN}^{35}$. This was found to be consistent with the previously calculated value from the $J_{\mathrm{a}}-V$ analysis using Eq. (5). Moreover, we analytically modeled the conduction using Eqs.(1)-(7) along with the extracted parameters from Table I and obtained a good fit with the $J_{\mathrm{a}}-V$ plot.

\section{Optical Measurements}

Infrared (IR) electroluminescence (EL) was observed from the fabricated $\mathrm{Al} / \mathrm{GaN} / \mathrm{p}-\mathrm{Si}$ (Fig 8(a)) diode. The measured spectrum (Fig $8(\mathrm{~b})$ ) is centered around the $1.12 \mu \mathrm{m}$ wavelength corresponding to the Si bandgap $\left(E_{\mathrm{g}-\mathrm{Si}}=1.12 \mathrm{eV}\right)$ with a full-width-halfmaximum $(\mathrm{FWHM}) \sim 96 \mathrm{meV}$ at $300 \mathrm{~K}$. The observed FWHM is in excess of $\sim 1.8 \mathrm{kT}^{43}$ because of light being emitted from silicon, an indirect band gap semiconductor where interaction with phonons during the radiative recombination process leads to the broadening of the EL-spectrum compared to that in direct band-gap semiconductors ${ }^{44,45}$. Both the peak wavelength and the FWHM are in good agreement when compared with standard p-n junctions in silicon ${ }^{46}$.

For reference sake, we also realized an $\mathrm{Al} / \mathrm{p}-\mathrm{Si}$ (with native oxide) diode ${ }^{47}$ without any poly-GaN layer in between. From this device, faint IR emission was observed which was too weak to be detected by our spectrometer as reported before ${ }^{47}$. This is also in line with the detected photocurrent $\left(I_{\mathrm{PD}}\right)$, Fig. 9(a), using an off-chip Si photodiode $(\mathrm{PD})^{14}$ which is a measure of the emission intensity corresponding to the injected diode current $\left(I_{\mathrm{LED}}\right)$. For the same $I_{\mathrm{LED}}$, the measured $I_{\mathrm{PD}}$ and therefore emission intensity for the $\mathrm{Al} / \mathrm{GaN} / \mathrm{p}$-Si diode $\left(I_{\mathrm{PD}} \sim 1 \mathrm{nA}\right)$ is $\sim$ 3 orders of magnitude higher than for the $\mathrm{Al} / \mathrm{p}-\mathrm{Si}$ diode $\left(I_{\mathrm{PD}} \sim 1 \mathrm{pA}\right)$. The emission intensity in the $\mathrm{GaN} / \mathrm{p}-\mathrm{Si}$ diode was also found to gradually increase with the injected power as expected from the LED behavior ${ }^{43}$.

Further, the $I_{\mathrm{PD}} / I_{\mathrm{LED}}$ ratio, is proportional to the internal quantum efficiency (IQE) of the LED. This is so because $I_{\mathrm{PD}} / I_{\mathrm{LED}}=\eta_{\mathrm{PD}} \cdot \eta_{\text {ext. }} \cdot \eta_{\mathrm{LED}}$, where $\eta_{\mathrm{PD} / \text { ext. } / \mathrm{LED}}$ is the IQE of detection in the $\mathrm{PD} /$ extraction efficiency of light/IQE of the LED, respectively ${ }^{46}$. Out of the three efficiencies, only $\eta_{\mathrm{LED}}$ is a function of $I_{\mathrm{LED}}$. Thus, the trend in $\eta_{\text {LED }}$ versus $I_{\text {LED }}$ is reflected in the trend in $I_{\mathrm{PD}} / I_{\mathrm{LED}}$ versus $I_{\mathrm{LED}}$. As shown in the Fig. $9(\mathrm{~b})$, in the low injection regime, $\eta_{\mathrm{LED}}$ was found to be increasing for higher $I_{\text {LED }}$ and no droop was observed up till an injected current density $\sim 25 \mathrm{~A} / \mathrm{cm}^{2}$.

Based on our measured $I_{\mathrm{PD}}$ (with a $\eta_{\mathrm{PD}}$ of 0.2 at $\sim$ $1120 \mathrm{~nm}$ free-space wavelength ${ }^{14}$ ), we have estimated the external quantum efficiency of our LED to be $3.5 \times 10^{-7}$. Further, from an estimate of the optical extraction efficiency $\left(\sim 1.5 \times 10^{-3}\right)$ of our measurement set-up ${ }^{48}$, we calculated the lower limit of the $\eta_{\mathrm{LED}}$ of our LED to be
$2 \times 10^{-4}$ without taking into account losses at the top electrode which blocks majority of emission, allowing only light from the periphery of the hexagonal openings (Fig 8(a)) to be detected.
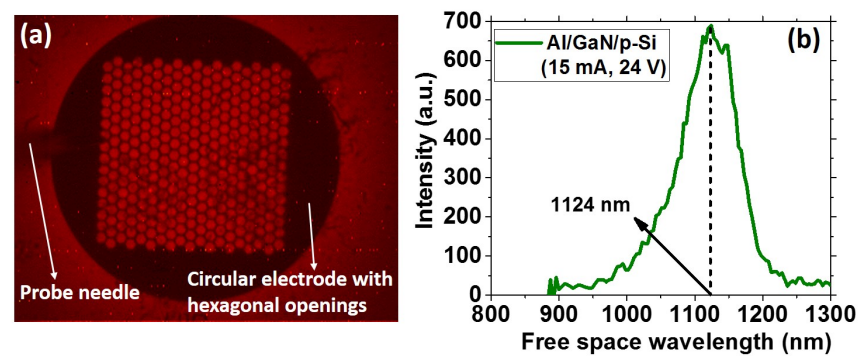

FIG. 8. (a) Bright field IR micrograph of the fabricated $\mathrm{GaN} / \mathrm{p}-\mathrm{Si}$ diode at a constant forward current drive of 15 $\mathrm{mA}\left(J=19 \mathrm{~A} / \mathrm{cm}^{2}\right)$, (b) Optical spectrum of the emitted light from the same diode.

The observed EL from the GaN/p-Si device is attributed to carrier recombination at the silicon side of the GaN/Si interface as illustrated in Fig. 3. No ultraviolet (UV) emission originating from the GaN band-edge was observed. We expect a negligible hole concentration in the GaN layer due to the high valence band offset and consequently no significant radiative recombination in the GaN layer. The role of the single type of charge carrier (i.e., electrons) in the conduction in GaN layer is also confirmed by the observed SCLC behavior. The improved emitter efficiency in case of GaN/Si diode could be attributed to the wide band gap of the GaN layer and resultant band discontinuity at the GaN/Si interface. Previously, enhanced emission from Si in the presence of native oxide has been reported ${ }^{49}$. However, in our case, no conclusive evidence of any possible role of the native oxide in the observed EL could be established. Both our diodes i.e. $\mathrm{Al} / \mathrm{GaN} / \mathrm{p}-\mathrm{Si}$ and $\mathrm{Al} / \mathrm{p}$-Si had a native oxide.

Fig. 10 shows measured $I-V$ characteristics of two different devices: the $\mathrm{Al} / \mathrm{p}-\mathrm{Si}$ and $\mathrm{Al} / \mathrm{GaN} / \mathrm{p}-\mathrm{Si}$ diode. The data indicate that for the same injected current level $I_{\text {LED }}$, as subjected to our EL measurements, the internal junction voltage $V_{\mathrm{j}}$ increases for devices with a reduced current level. As a result, $V_{\mathrm{j}-\mathrm{pSi}}<V_{\mathrm{j}-\mathrm{pSiGaN}}$. Therefore, the product of the hole and electron density (i.e., $p$. $n$ ) in $\mathrm{Si}$ (light emitting region in both the cases) which is exponentially dependent on $V_{\mathrm{j}}$, also follows the same order $\left(p \cdot n_{\mathrm{pSi}}<p \cdot n_{\mathrm{pSiGaN}}\right)$. This explains the enhanced emission from the GaN/Si diode compared to that of the Si-only diode, since a higher $p \cdot n$-value yields a higher radiative recombination rate (i.e., light emission) $)^{50}$.

\section{Discussion}

From the analysis discussed before it can be concluded that the current flow in our ALD grown thin-film polyGaN/Si diode is largely limited by the GaN layer. This 

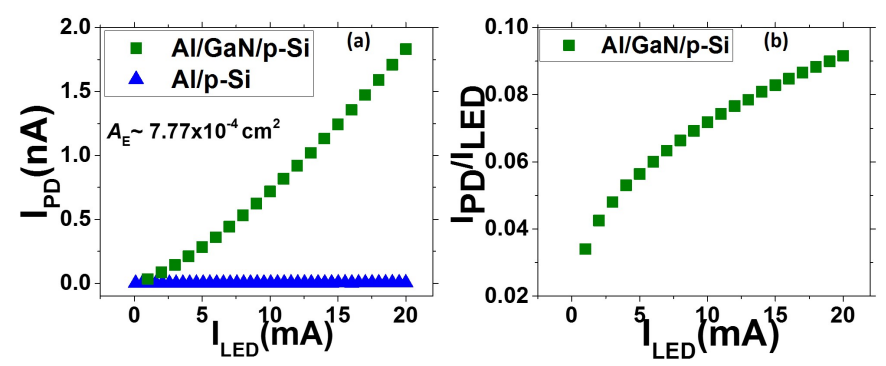

FIG. 9. (a) Measured short-circuit current $\left(I_{\mathrm{PD}}\right)$ in the photodiode $(\mathrm{PD})$ versus injected forward current $\left(I_{\mathrm{LED}}\right)$ for the $\mathrm{Al} / \mathrm{GaN} / \mathrm{p}-\mathrm{Si}$ diode and $\mathrm{Al} / \mathrm{p}-\mathrm{Si} \operatorname{diode}^{47}$. (b) $I_{\mathrm{PD}} / I_{\mathrm{LED}}$ $\left(\propto \eta_{\text {LED }}\right)$ versus $I_{\text {LED }}$.

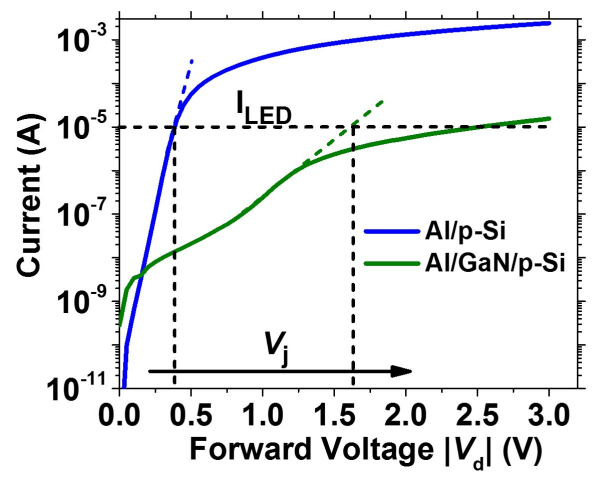

FIG. 10. Measured I-V characteristics of $\mathrm{Al} / \mathrm{GaN} / \mathrm{p}-\mathrm{Si}$ diode and $\mathrm{Al} / \mathrm{p}-\mathrm{Si}$ diode.

allowed us to estimate the basic electrical properties of the polycrystalline layer via $I-V$ analysis using the single shallow trap SCLC model ${ }^{23}$. The relatively large trap density in the unintentionally doped thin film of the wide band gap material could be the reason for the observed SCLC behavior. However, in a good heterojunction ${ }^{51}$, for electronic applications such as bipolar transistors, the current should be limited by diffusion in the narrow band gap material.

Despite this, the optical measurements indicate that such engineered poly-GaN based heterojunctions, realized at low temperature, could be interesting for fabricating efficient Si based IR emitters using a relatively simple process. Such heterojunctions can also be investigated for Si based solar cells with an improved injector efficiency ${ }^{30,52,53}$. Heterojunction diodes utilizing wide bandgap materials as an emitter are interesting for enhancing the injector efficiency, for example, of the electroluminescent diode ${ }^{54}$ and has been reported before for different materials ${ }^{49,55-59}$.For example, Favennec et al. ${ }^{55}$ reported the improved luminescence from Er when implanted in a wide band gap materials matrix. More recently, near IR emission peaking at 826 $\mathrm{nm}$ from $\mathrm{GaN} / \mathrm{Si}$ heterojunctions was also reported by Han et al. ${ }^{59,60}$, where unlike in our process, a $500 \mathrm{~nm}$ thick GaN nanocrystalline film was grown at $1050{ }^{\circ} \mathrm{C}$ with a CVD process on an Si nanoporous pillar array (NPA). The difference in the observed peak EL wavelength with that obtained from our $\mathrm{GaN} / \mathrm{Si}$ devices is due to the reported higher energy bandgap $\left(E_{\mathrm{g}-\mathrm{Si}-\mathrm{NPA}}=2\right.$ $\mathrm{eV})$ and electron affinity $\left(\chi_{\mathrm{Si}-\mathrm{NPA}}=3.6 \mathrm{eV}\right)$ of the Si NPA and therefore resulting in different band alignment and heterojunction properties. Interestingly, the conduction mechanism reported for the GaN/Si-NPA device ${ }^{60}$ was also attributed to an SCLC process.

\section{CONCLUSIONS}

We showed that heterojunction diodes can be realized at a low temperature by adopting ALD of poly-GaN on an Si wafer. The fabricated heterojunction devices showed good rectification and also infrared EL. The current transport in the forward bias is explained by SCLC in the presence of traps as possible dominant transport mechanism. Following the single trap SCLC model along with temperature dependent $J-V$ analysis, a minimum trap density of $4.6 \times 10^{17} / \mathrm{cm}^{3}$ at an energy level $0.13 \mathrm{eV}$ below the conduction band minimum was estimated in the polycrystalline film. Other electrical properties of the poly-GaN layer such as free carrier concentration, effective density of states in the conduction band, electron mobility and dielectric relaxation time were also determined using the SCLC analysis. The observed IR emission from EL is attributed to band-to-band recombination in the Si near the heterojunction interface because of a high $p \cdot n$-value. The presence of a thin polycrystalline GaN significantly improves the IR emission. The introduction of such engineered heterostructures could be a way forward for making efficient optoelectronic devices such as IR emitters and solar cells realized in bulk Si. The study of such thin-film ALD GaN on Si is also promising for future nanoscale (opto)electronics based on this platform.

\section{ACKNOWLEDGMENTS}

The authors acknowledge partial financial support by the NWO Domain Applied and Engineering Sciences (TTW), The Netherlands (OTP 2014, under Project 13145).

${ }^{1}$ S. Strite and H. Morkoç, "GaN, AlN, and InN: a review," Journal of Vacuum Science \& Technology B: Microelectronics and Nanometer Structures Processing, Measurement, and Phenomena 10, 1237-1266 (1992).

${ }^{2}$ L. F. Eastman and U. K. Mishra, "The toughest transistor yet [GaN transistors]," IEEE spectrum 39, 28-33 (2002).

${ }^{3} \mathrm{H}$. Morkoç, Handbook of nitride semiconductors and devices, $M a-$ terials Properties, Physics and Growth, Vol. 1 (John Wiley \& Sons, 2009).

${ }^{4}$ D. Zhu, D. Wallis, and C. Humphreys, "Prospects of III-nitride optoelectronics grown on Si," Reports on Progress in Physics 76, 106501 (2013). 
${ }^{5}$ M. Ishida, T. Ueda, T. Tanaka, and D. Ueda, "GaN on Si technologies for power switching devices," IEEE Transactions on Electron Devices 60, 3053-3059 (2013).

${ }^{6}$ A. Dadgar, "Sixteen years GaN on Si," Physica Status Solidi (b) 252, 1063-1068 (2015).

${ }^{7}$ S. Jain, M. Willander, J. Narayan, and R. V. Overstraeten, "IIInitrides: Growth, characterization, and properties," Journal of Applied Physics 87, 965-1006 (2000).

${ }^{8}$ N. Biyikli and A. Haider, "Atomic layer deposition: an enabling technology for the growth of functional nanoscale semiconductors," Semiconductor Science and Technology 32, 093002 (2017). ${ }^{9}$ C. Ozgit-Akgun, E. Goldenberg, A. K. Okyay, and N. Biyikli, "Hollow cathode plasma-assisted atomic layer deposition of crystalline $\mathrm{AlN}, \mathrm{GaN}$ and $\mathrm{Al} \times \mathrm{Ga} 1-\mathrm{x} \mathrm{N}$ thin films at low temperatures," Journal of Materials Chemistry C 2, 2123-2136 (2014).

${ }^{10}$ N. Karam, T. Parodos, P. Colter, D. McNulty, W. Rowland, J. Schetzina, N. El-Masry, and S. M. Bedair, "Growth of device quality GaN at $550 \mathrm{C}$ by atomic layer epitaxy," Applied Physics Letters 67, 94-96 (1995).

${ }^{11} \mathrm{~S}$. Bolat, C. Ozgit-Akgun, B. Tekcan, N. Biyikli, and A. Okyay, "Low temperature thin film transistors with hollow cathode plasma-assisted atomic layer deposition based GaN channels," Applied Physics Letters 104, 243505 (2014).

${ }^{12}$ S. Bolat, B. Tekcan, C. Ozgit-Akgun, N. Biyikli, and A. K. Okyay, "Electronic and optical device applications of hollow cathode plasma assisted atomic layer deposition based gan thin films," Journal of Vacuum Science \& Technology A: Vacuum, Surfaces, and Films 33, 01A143 (2015).

${ }^{13} \mathrm{~S}$. Bolat, Z. Sisman, and A. Okyay, "Demonstration of flexible thin film transistors with GaN channels," Applied Physics Letters 109, 233504 (2016).

${ }^{14}$ K. Mok, L. Qi, A. Vlooswijk, and L. K. Nanver, "Self-aligned two-layer metallization with low series resistance for litho-less contacting of large-area photodiodes," Solid-State Electronics 111, 210-217 (2015).

${ }^{15}$ G. Yu, G. Wang, H. Ishikawa, M. Umeno, T. Soga, T. Egawa, J. Watanabe, and T. Jimbo, "Optical properties of wurtzite structure GaN on sapphire around fundamental absorption edge $(0.78-4.77 \mathrm{eV})$ by spectroscopic ellipsometry and the optical transmission method," Applied Physics Letters 70, 3209-3211 (1997).

${ }^{16}$ J. Hedman and N. Mårtensson, "Gallium nitride studied by electron spectroscopy," Physica Scripta 22, 176 (1980).

${ }^{17}$ Sentaurus TCAD, Synopsys Inc., Mountain View, CA, USA, version l-2016.03 ed. (2016).

${ }^{18} \mathrm{~V}$. Bermudez, "Study of oxygen chemisorption on the GaN (0001)-(1× 1) surface," Journal of Applied Physics 80, 11901200 (1996).

${ }^{19} \mathrm{C}$. Wu and A. Kahn, "Investigation of the chemistry and electronic properties of metal/gallium nitride interfaces," Journal of Vacuum Science \& Technology B: Microelectronics and Nanometer Structures Processing, Measurement, and Phenomena 16, 2218-2223 (1998).

${ }^{20} \mathrm{H}$. Tampo, T. Yamanaka, K. Yamada, K. Ohnishi, M. Hashimoto, and H. Asahi, "Field emission from polycrystalline GaN grown on Mo substrate," Japanese Journal of Applied Physics 41, L907 (2002).

${ }^{21}$ T. Yamashita, S. Hasegawa, S. Nishida, M. Ishimaru, Y. Hirotsu, and H. Asahi, "Electron field emission from GaN nanorod films grown on Si substrates with native silicon oxides," Applied Physics Letters 86, 082109 (2005).

${ }^{22}$ A. Rose, "Space-charge-limited currents in solids," Physical Review 97, 1538 (1955).

${ }^{23}$ M. A. Lampert and P. Mark, "Current injection in solids," Academic Press Inc., New York , 3 (1970).

${ }^{24}$ J. Vesely, M. Shatzkes, and P. Burkhardt, "Space-charge-limited current flow in gallium nitride thin films," Physical Review B 10, 582 (1974).

${ }^{25}$ X. Shen, D. Zhao, Z. Liu, Z. Hu, H. Yang, and J. Liang, "Spacecharge-limited currents in GaN Schottky diodes," Solid-State
Electronics 49, 847-852 (2005).

${ }^{26} \mathrm{H}$. Hall, M. Awaah, and K. Das, "Deep-level dominated rectifying contacts for n-type GaN films," Physics Status Solidi (a) 201, 522-528 (2004).

${ }^{27}$ A. A. Talin, F. Léonard, B. Swartzentruber, X. Wang, and S. D. Hersee, "Unusually strong space-charge-limited current in thin wires," Physical Review Letters 101, 076802 (2008).

${ }^{28}$ M. Kumar, T. N. Bhat, M. K. Rajpalke, B. Roul, N. Sinha, A. Kalghatgi, and S. Krupanidhi, "Negative differential capacitance in n-GaN/p-Si heterojunctions," Solid State Communications 151, 356-359 (2011).

${ }^{29}$ M. Kumar, B. Roul, T. N. Bhat, M. K. Rajpalke, A. Kalghatgi, and S. Krupanidhi, "Carrier-transport studies of III-nitride/Si3N4/Si isotype heterojunctions," Physica Status Solidi (a) 209, 994-997 (2012)

${ }^{30}$ K. Saron, M. Hashim, N. Naderi, and N. K. Allam, "Interface properties determined the performance of thermally grown GaN/Si heterojunction solar cells," Solar Energy 98, 485-491 (2013).

${ }^{31}$ R. Ghosh and D. Basak, "Electrical and ultraviolet photoresponse properties of quasialigned $\mathrm{ZnO}$ nanowires/p-Si heterojunction," Applied Physics Letters 90, 243106 (2007).

${ }^{32}$ J. Ye, S. Gu, S. Zhu, W. Liu, S. Liu, R. Zhang, Y. Shi, and Y. Zheng, "Electroluminescent and transport mechanisms of n$\mathrm{ZnO} /$ p-Si heterojunctions," Applied Physics Letters 88, 182112 (2006).

${ }^{33}$ Y. I. Alivov, J. Van Nostrand, D. C. Look, M. Chukichev, and B. Ataev, "Observation of $430 \mathrm{~nm}$ electroluminescence from $\mathrm{ZnO} / \mathrm{GaN}$ heterojunction light-emitting diodes," Applied Physics Letters 83, 2943-2945 (2003).

${ }^{34}$ F.-C. Chiu, H.-W. Chou, and J. Y.-m. Lee, "Electrical conduction mechanisms of metal/ La2O3/ Si structure," Journal of Applied Physics 97, 103503 (2005).

${ }^{35}$ M. E. Levinshtein, S. L. Rumyantsev, and M. S. Shur, Properties of Advanced Semiconductor Materials: GaN, AIN, InN, BN, SiC, SiGe (John Wiley \& Sons, 2001).

${ }^{36}$ G. Roberts and F. W. Schmidlin, "Study of localized levels in semi-insulators by combined measurements of thermally activated ohmic and space-charge-limited conduction," Physical Review 180, 785 (1969)

${ }^{37}$ J. Fedison, T. Chow, H. Lu, and I. Bhat, "Electrical characteristics of magnesium-doped gallium nitride junction diodes," Applied Physics Letters 72, 2841-2843 (1998).

${ }^{38}$ E. Suzuki, D. K. Schroder, and Y. Hayashi, "Carrier conduction in ultrathin nitrided oxide films," Journal of Applied Physics 60, 3616-3621 (1986).

${ }^{39}$ M. P. Houng, Y. H. Wang, and W. J. Chang, "Current transport mechanism in trapped oxides: A generalized trap-assisted tunneling model," Journal of Applied Physics 86, 1488-1491 (1999).

${ }^{40}$ O. Blank, H. Reisinger, R. Stengl, M. Gutsche, F. Wiest, V. Capodieci, J. Schulze, and I. Eisele, "A model for multistep trap-assisted tunneling in thin high-k dielectrics," Journal of Applied Physics 97, 044107 (2005).

${ }^{41}$ O. Mitrofanov and M. Manfra, "Poole-Frenkel electron emission from the traps in AlGaN/GaN transistors," Journal of Applied Physics 95, 6414-6419 (2004).

${ }^{42}$ E. Arslan, S. Bütün, and E. Ozbay, "Leakage current by Frenkel-Poole emission in Ni/Au Schottky contacts on $\mathrm{Al} 0.83$ In $0.17 \mathrm{~N} / \mathrm{AlN} / \mathrm{GaN}$ heterostructures," Applied Physics Letters 94, 142106 (2009).

${ }^{43}$ E. F. Schubert, T. Gessmann, and J. K. Kim, Light emitting diodes (Wiley Online Library, 2005).

${ }^{44}$ A. Fiory and N. Ravindra, "Light emission from silicon: Some perspectives and applications," Journal of Electronic Materials 32, 1043-1051 (2003).

${ }^{45}$ T.-H. Cheng, Y. Chu-Su, C.-S. Liu, and C.-W. Lin, "Phononassisted transient electroluminescence in Si," Applied Physics Letters 104, 261102 (2014).

${ }^{46}$ S. Dutta, V. Agarwal, R. J. E. Hueting, J. Schmitz, and A.-J. Annema, "Monolithic optical link in silicon-on-insulator CMOS 
technology," Optics Express 25, 5440-5456 (2017)

${ }^{47}$ G. Gupta, S. Dutta, S. Banerjee, and R. J. E. Hueting, "Minority Carrier Injection in High-Barrier Si-Schottky Diodes," IEEE Transactions on Electron Devices 65, 1276-1282 (2018).

${ }^{48}$ S. Dutta, G. J. Wienk, R. J. E. Hueting, J. Schmitz, and A.-J. Annema, "Optical Power Efficiency Versus Breakdown Voltage of Avalanche-Mode Silicon LEDs in CMOS," IEEE Electron Device Letters 38, 898-901 (2017).

${ }^{49}$ G. Qin, Y. Huang, J. Lin, L. Zhang, B. Zong, and B. Zhang, "Electroluminescence from $\mathrm{Au} /$ native oxide/p-Si and its correlation to that from $\mathrm{Au}$ /porous Si," Solid State Communications 94, 607-612 (1995).

${ }^{50}$ V. Puliyankot and R. J. E. Hueting, "One-dimensional physical model to predict the internal quantum efficiency of Si-based LEDs," IEEE Transactions on Electron Devices 59, 26-34 (2012).

${ }^{51} \mathrm{H}$. Kroemer, "Two integral relations pertaining to the electron transport through a bipolar transistor with a nonuniform energy gap in the base region," Solid-State Electronics 28, 1101-1103 (1985).

52 J. Wu, W. Walukiewicz, K. Yu, W. Shan, J. Ager Iii, E. Haller, H. Lu, W. J. Schaff, W. Metzger, and S. Kurtz, "Superior radiation resistance of In 1- x Ga x N alloys: full-solar-spectrum photovoltaic material system," Journal of Applied Physics 94, 6477-6482 (2003).

${ }^{53}$ Y. Tang, Z. Chen, H. Song, C. Lee, H. Cong, H. Cheng, W. Zhang, I. Bello, and S. Lee, "Vertically aligned p-type singlecrystalline GaN nanorod arrays on n-type Si for heterojunction photovoltaic cells," Nano Letters 8, 4191-4195 (2008).

${ }^{54}$ S. M. Sze and K. K. Ng, Physics of semiconductor devices (John wiley \& sons, 2006).

${ }^{55}$ P. Favennec, H. L'haridon, M. Salvi, D. Moutonnet, and Y. Le Guillou, "Luminescence of erbium implanted in various semiconductors: IV, III-V and II-VI materials," Electronics Letters 25, 718-719 (1989)

${ }^{56}$ M. A. Lourenço, M. A. Hughes, K. T. Lai, I. M. Sofi, W. Ludurczak, L. Wong, R. M. Gwilliam, and K. P. Homewood, "SiliconModified Rare-Earth Transitions-A New Route to Near-and MidIR Photonics," Advanced Functional Materials 26, 1986-1994 (2016).

${ }^{57}$ A. Steckl, M. Garter, R. Birkhahn, and J. Scofield, "Green electroluminescence from Er-doped GaN Schottky barrier diodes," Applied Physics Letters 73, 2450-2452 (1998).

${ }^{58}$ J. B. You, X. W. Zhang, S. G. Zhang, J. X. Wang, Z. G. Yin, H. R. Tan, W. J. Zhang, P. K. Chu, B. Cui, A. M. Wowchak, A. M. Dabiran, and P. P. Chow, "Improved electroluminescence from n-ZnO/AlN/p-GaN heterojunction light-emitting diodes," Applied Physics Letters 96, 201102 (2010).

${ }^{59}$ C. B. Han, C. He, and X. J. Li, "Near-Infrared Light Emission from a GaN/Si Nanoheterostructure Array," Advanced Materials 23, 4811-4814 (2011).

${ }^{60}$ C. B. Han, C. He, X. B. Meng, Y. R. Wan, Y. T. Tian, Y. J. Zhang, and X. J. Li, "Effect of annealing treatment on electroluminescence from GaN/Si nanoheterostructure array," Optics Express 20, 5636-5643 (2012). 